\title{
Some interesting freshwater algae from Chimdi lake including a new record for Nepal
}

\author{
Shiva Kumar Rai ${ }^{*}$ and Ram Kumar Rai ${ }^{2}$ \\ ${ }^{1}$ Department of Botany, Post Gradaute Campus, T.U., Biratnagar, Nepal \\ ${ }^{2}$ Department of Botany, MMAM Campus, T.U., Biratnagar, Nepal \\ *E-mail: sk.khaling@gmail.com
}

\begin{abstract}
A preliminary study on algae of Chimdi lake was carried out. A total six freshwater algae viz., Oscillatoria splendida Grev. ex Gom., Cylindrospermum stagnale (Kuetz.) Born. et Flah. f. variabilis Prasad, Gloeotrichia raciborskii Woloszynska var. kashiense Rao, Melosira varians Ag., Crucigenia crucifera (Wolle) Collins and Euastrum spinulosum Delp. var. bellum Scott et Prescott were recorded from Chimdi lake. Euastrum spinulosum var. bellum was recorded for the first time from Nepal.
\end{abstract}

Key words: Freshwater algae, taxonomy and diversity, Chimdi lake, Oscillatoria, Nepal

\section{Introduction}

Algae grow in water and act as primary producer, supplying energy for all aquatic organisms through food chain. Recent activities and researches on algal biofuel, algal super food, space food, genetic engineering, pollution control, bio-indicator, algal nanotechnology etc. are blooming fields in the Science, all based on the taxonomy of algae.

Topography of Nepal is as such that generally all types of world's climates are found within the small area and the country has rich lentic water resources like lakes, ponds, ditches etc. The lakes and ponds of Nepal harbor luxuriant growth of diverse species of algae. But, literature reveal that the algae of lentic environment of Nepal has not been studied extensively except sporadic works. Hickel (1973), Nakanishi et al. (1984) and Ishida (1986) have studied algae of Phewa lake; Watanabe (1995) has studied algae of Rara lake; Rai et al. (2008) has studied algae of Bees hazaar Tal; and Rai $(2005,2009,2011)$ has studied algae of Maipokhari and Betana pond. In the present study, an attempt has been made to study the algae of Chimdi lake, Nepal.

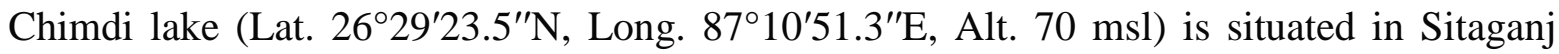
VDC, Sunsari district, about $12 \mathrm{~km}$ west from Biratnagar (Fig. 1). It has a total area of approximately 101.6 ha. The lake has permanent, clean and unpolluted water. The physicochemical parameters of Chimdi lake is as follows: water temperature ranges from $18.8^{\circ} \mathrm{C}$ (January) to $30.5^{\circ} \mathrm{C}$ (September), $\mathrm{pH}$ from 6.05 (April) to 8 (May), transparency from 2.13 $\mathrm{cm}$ (July) to $27.83 \mathrm{~cm}$ (September), dissolved oxygen from $4.82 \mathrm{mg} / \mathrm{l}$ (June) to $19.92 \mathrm{mg} / \mathrm{l}$ (September), total alkalinity from $32 \mathrm{mg} / 1$ (July) to $86 \mathrm{mg} / \mathrm{l}$ (March) and total hardness from $38 \mathrm{mg} / \mathrm{l}$ (June) to $140 \mathrm{mg} / \mathrm{l}$ (March) (Surana et al., 2010). It is inhabited by many macrophytes, viz., Echinochloa sp., Ipomoea carnea, Hydrilla verticillata, etc and is also famous for migratory birds. 


\section{Materials and Methods}

Algal samples were collected from the peripheral edge of Chimdli lake. Planktonic forms were collected with the help of plankton net and epiphytic forms were collected by squeezing submerged macrophytes. Samples were preserved in 4\% formaldehyde solution, tagged appropriately and brought to the Algae Research Lab, Department of Botany, P.G. Campus, Biratnagar for further investigation. Identification of algae was done with the help of Olympus CH20i Microscope following relevant literature. Classification and distribution of algae followed online website 'algaebase' (Guiry \& Guiry, 2012). Diatoms were identified after clearing the frustules following modified method of Patrick and Reimer (1966). Scale bar drawn in each algae picture is equal to $10 \mu \mathrm{m}$.

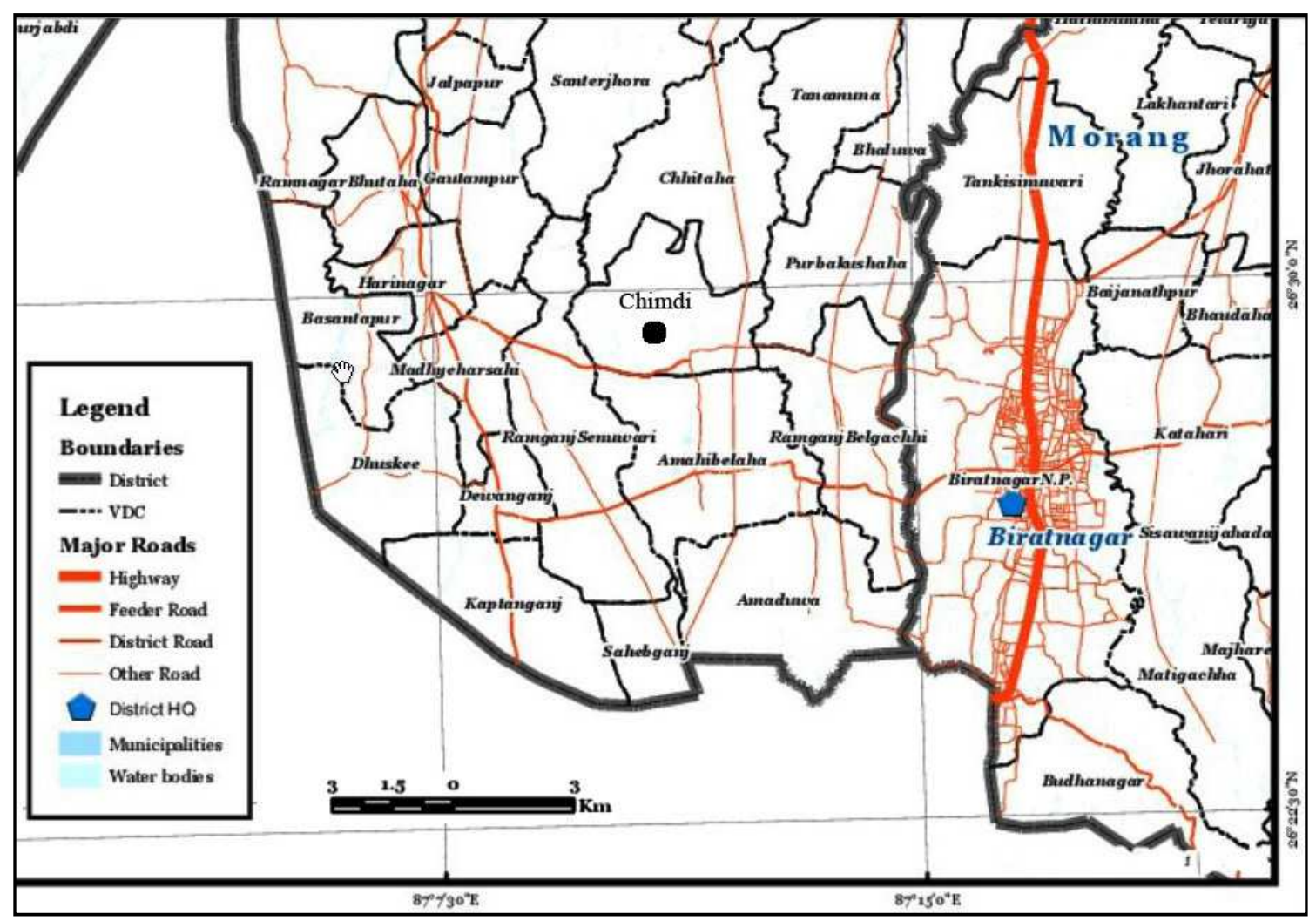

Figure 1. Map of the study area (Chimdi lake area).

\section{Taxonomic Description}

Phylum: Cyanobacteria

Class: Cyanophyceae

Subclass: Oscillatoriophycideae

Order: Oscillatoriales

Family: Oscillatoriaceae

Genus: Oscillatoria Vaucher 1803

1. Oscillatoria splendida Grev. ex Gom. (Pl. 1)

Current accepted name: Geitlerinema splendidum (Greville ex Gomont) Anagnostidis Lectotype: Oscillatoria princeps Vaucher ex Gomont. 
Taxonomic characters: Dark blue-green thin masses or scattered; trichome straight or bent, $2.5 \mu \mathrm{m}$ broad, without constrictions but with somewhat granulated cross-walls, gradually tapering at apex, apex often twisted or bent; cells 4-5 $\mu \mathrm{m}$ long; end cell $12 \mu \mathrm{m}$ long, capitates, nearly rounded.

Collection number and date: Ch 32 (10-02-2011).

Distribution in Nepal: Lirum glacier, 3,900 m, Rasuwa (Hirano, 1969); Chimdi lake, 73 m, Sunsari (Rai \& Misra, 2010).

World distribution: Britain (Whitton et al., 1998), Israel (Vinogradova et al., 2000), Queensland (Day et al., 1995), Romania (Caraus, 2012), Spain (Aboal, 1989), Sweden (Skuja, 1948).

Phylum: Cyanobacteria

Class: Cyanophyceae

Subclass: Nostocophycideae

Order: Nostocales

Family: Nostocaceae

Genus: Cylindrospermum Kützing 1843

2. Cylindrospermum stagnale (Kuetz.) Born. et Flah. f. variabilis Prasad (Pl. 2)

Holotype: Cylindrospermum majus Kützing.

Taxonomic characters: Light blue-green mat; trichomes single, cylindrical, slightly constricted at the cross-wall, 4-5 $\mu \mathrm{m}$ broad; cells 4-6 $\mu \mathrm{m}$ long; heterocyst single, cylindrical, sub elliptical or ellipsoidal, $12 \mu \mathrm{m}$ long, $6.5-7 \mu \mathrm{m}$ broad; akinet single, sub-cylindrical, elongate, $10 \mu \mathrm{m}$ long, $6.5 \mu \mathrm{m}$ broad.

Remarks: Present specimen has small spores.

Collection number and date: Ch 22 (10-02-2011).

Distribution in Nepal: Chimdi lake, 73 m, Sunsari (Rai \& Misra, 2010).

Phylum: Cyanobacteria

Phylum: Cyanobacteria

Class: Cyanophyceae

Subclass: Nostocophycideae

Order: Nostocales

Family: Rivulariaceae

Genus: Gloeotrichia Agardh 1842

3. Gloeotrichia raciborskii Woloszynska var. kashiense Rao (P1. 4)

Holotype: Gloeotrichia pisum (C. Agardh) Thuret ex Bornet \& Flhault.

Taxonomic characters: Trichome slightly constricted at cross-wall, 7-10 $\mu \mathrm{m}$ broad at base, 6-6.5 $\mu \mathrm{m}$ broad higher up; cell 7.5-9 $\mu \mathrm{m}$ long at base, upto $10 \mu \mathrm{m}$ long higher up; heterocyst single, spherical or ellipsoidal, $12.5 \mu \mathrm{m}$ long, $10-11.3 \mu \mathrm{m}$ broad; akinet ellipsoidal, outer wall smooth hyaline, $30 \mu \mathrm{m}$ long, $14.6 \mu \mathrm{m}$ broad (30-45 $\mu \mathrm{m}$ broad with sheath).

Collection number and date: Ch 34 (10-02-2011). 
Distribution in Nepal: Chimdi lake, 73 m, Sunsari (Rai \& Misra, 2010).

Phylum: Ochrophyta

Class: Bacillariophyceae

Subclass: Coscinodiscophycidae

Superorder: Coscinodiscanae

Order: Melosirales

Family: Melosiraceae

Genus: Melosira C.A. Agardh 1824

4. Melosira varians Ag. (Pl. 3)

Holotype: Melosira nummuloides C. Agardh

Homotypic synonymous: Lysigonium varians (C. Agardh) De Toni 1892

Taxonomic characters: Frustule cylindrical, semicell $12 \mu \mathrm{m}$ long, $15 \mu \mathrm{m}$ in diameter; generally united into a straight or rarely curved chains, valves more or less convex with fine punctae interspersed with coarse dots; girdle finely punctate, slightly smooth.

Collection number and date: Ch 30 (10-02-2011).

Distribution in Nepal: A pond at Ankhu Khola, $640 \mathrm{~m}$ and Luitel Bhanjyang, $770 \mathrm{~m}$, Gorkha (Hirano, 1955); a small pond near Pitchhara Nahar, 73 m, Biratnagar (Rai \& Rai, 2005); Lamphengwa river, Gajurmukhi VDC, Ilam (Rai et al., 2008); Muga river at Pakhribas Agriculture Research Centre, $1850 \mathrm{~m}$, Dhankuta and Hongchur river, $900 \mathrm{~m}$, Khotang (Misra et al., 2009); Betana wetland, 123 m, Belbari VDC, Morang (Rai, 2011).

World distribution: Argentina (Rodriguez et al., 2006), Brazil (Eskinazi-Leça et al., 2010), Britain (Whitton et al., 1998), China (Hu \& Wei, 2006), Germany (Bahulikar \& Kroth, 2007), Hawaiian Islands (Sherwood, 2004), Iran (Jamaloo et al., 2006), Israel (Tsarenko et al., 2000), New South Wales (Day et al., 1995), Queensland (Bostock \& Holland, 2010), Romania (Caraus, 2012), South Australia (Day et al., 1995), Spain (Pérez et al., 2010), Turkey (Ersanli \& Gönülol, 2006), Victoria (Roberts et al., 2004).

Phylum: Chlorophyta

Subphylum: Tetraphytina

Class: Trebouxiophyceae

Order: Trebouxiophyceae ordo incertae sedis

Family: Trebouxiophyceae incertae sedis

Genus: Crucigenia Morren 1830

\section{Crucigenia crucifera (Wolle) Collins (Pl. 5)}

Holotype: Crucigenia quadrata Morren.

Synonym: Crucigeniella crucifera (Wolle) Komárek

Taxonomic characters: Planktonic; coenobia 4-celled, rhomboidal, with rounded ends, slightly concave sides, central rectangular space, 24-27.5 $\mu \mathrm{m}$ in diameter, sometimes 4 colonies jointed to form multiple colonies; cell elongate, outer face concave, inner attached margin concave or slightly convex, 7.5 $\mu \mathrm{m}$ long, 4-5 $\mu \mathrm{m}$ broad; chloroplast single, parietal, laminate, without a pyrenoid. 

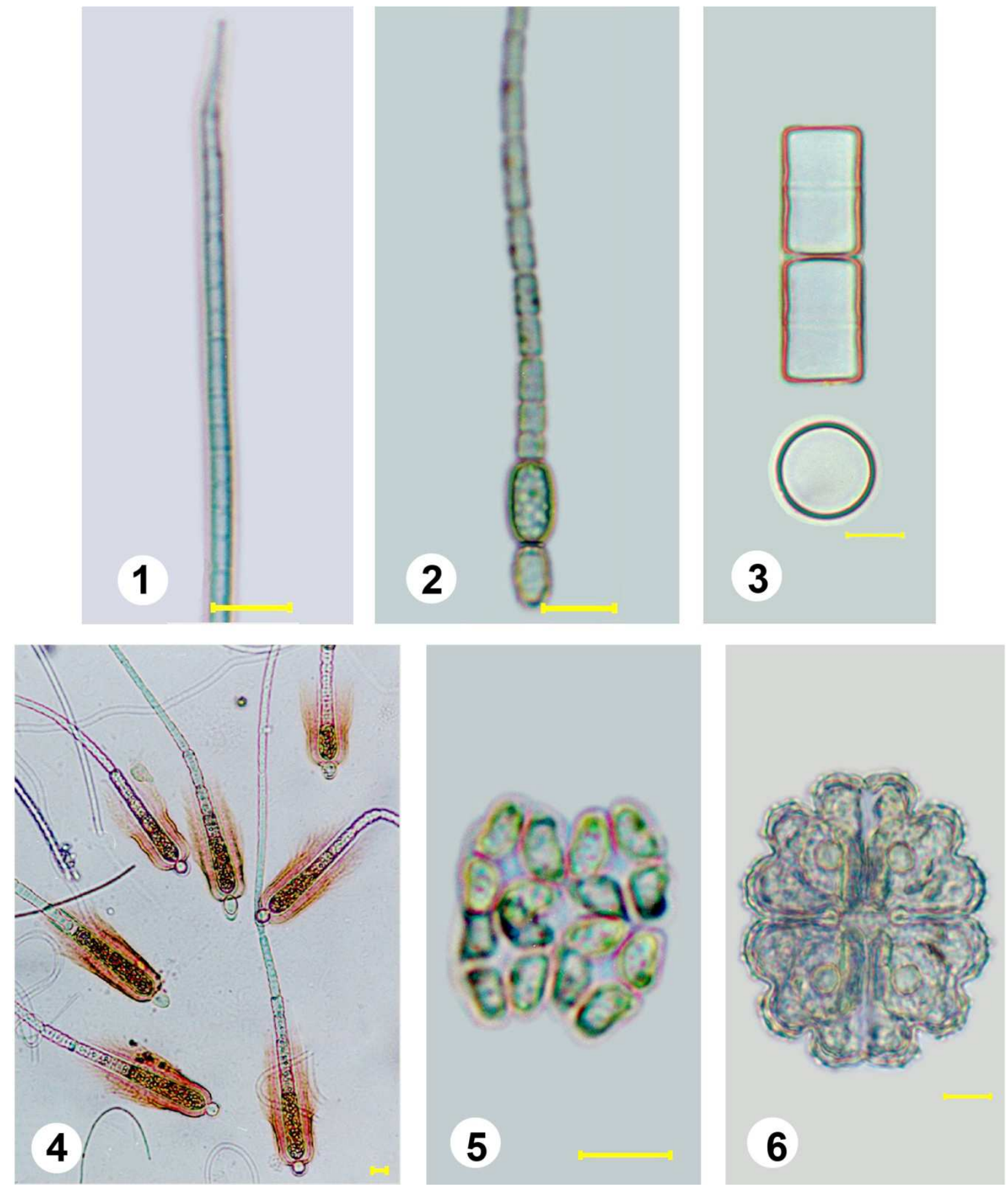

Plate 1. Oscillatoria splendida Grev. ex Gom., Plate 2. Cylindrospermum stagnale (Kuetz.) Born. et Flah. f. variabilis Prasad, Plate 3. Melosira varians Ag., Plate 4. Gloeotrichia raciborskii Woloszynska var. kashiense Rao, Plate 5. Crucigenia crucifera (Wolle) Collins, Plate 6. Euastrum spinulosum Delp. var. bellum Scott et Prescott. (Scale bar in each plate $=10$ $\mu \mathrm{m})$ 
Ecology: Usually found in the meso to eutrophic rivers, ponds, lakes and reservoirs.

Collection number and date: Ch 35 (10-02-2011).

Distribution in Nepal: Rupa lake, 900 m, Kaski (Ishida, 1986); Fish pond as Hetauda, Makawanpur (Sahay et al., 1992).

World distribution: Asia: Taiwan (Anonymous, 2012).

Phylum: Charophyta

Class: Zygnematophyceae

Order: Desmidiales

Family: Desmidiaceae

Genus: Euastrum Ehrenberg 1832

\section{Euastrum spinulosum Delp. var. bellum Scott et Prescott (P1. 6)}

Taxonomic characters: Cell large, 62-63 $\mu \mathrm{m}$ long, $52.5 \mu \mathrm{m}$ broad, longer than broad, deeply constricted; sinus narrow and linear; semi cell 5 lobed, lobes widely rounded with acute indentations between them, lateral lobes furnished with 5-6 spines, polar lobes broadly truncate with a median notch, polar lobes $23 \mu \mathrm{m}$ broad; verrucae of the central rosettes unusually large; isthmus $13-15 \mu \mathrm{m}$ wide.

Collection number and date: Ch 28 (10-02-2011).

Distribution in Nepal: New record for Nepal.

World distribution: Asia: Taiwan (Anonymous, 2012).

\section{Acknowledgements}

Authors would like to acknowledge the Head, Department of Botany, P.G. Campus, T.U., Biratnagar for laboratory facility. Thanks are also due to Prof. Dr. P.K. Misra, University of Lucknow for microphotography facility. The assistance of local people of Chimdi VDC during algae collection was greatly acknowledgeable.

\section{References}

Aboal, M. 1989. Flora algal del río Benamor (Cuenca del Segura, SE de España). Limnética 5: $1-11$.

Anonymous. 2012. Biota Taiwanica. Algae of Taiwan. Algae of Taiwan Checklist. http://algae.biota. biodiv.tw/catalog browse on 16th Sept., 2012.

Bahulikar, R.A. \& P.G. Kroth 2007. Localization of EPS components secreted by freshwater diatoms using differential staining with fluorophore-conjugated lectins and other fluorochromes. European Journal of Phycology 42(2): 199-208.

Bostock, P.D. \& A.E. Holland. 2010. Census of the Queensland Flora. Brisbane: Queensland Herbarium Biodiversity and Ecosystem Sciences, Department of Environment and Resource Management. pp. 1-320.

Caraus, I. 2012. Algae of Romania. A distributional checklist of actual algae. Version 2.3 third revision. Bacau: Univ. Bacau.

Day, S.A., R.P. Wickham, T.J. Entwisle \& P.A. Tyler. 1995. Bibliographic check-list of nonmarine algae in Australia. Flora of Australia Supplementary Series 4: 276. 
Ersanli, E. \& A. Gönülol. 2006. A study on the phytoplankton of Lake Simenit, Turkey. Cryptogamie Algologie 27: 289-305.

Eskinazi-Leça, E., M. da G. Gonçalves da Silva Cunha, M.F. Santiago, G.C. Palmeira Borges, J.M. Cabral de Lima, M.H. Da Silva, J. De Paula Lima \& M. Menezes. 2010. Bacillariophyceae. In: Catálogo de plantas e fungos do Brasil, Vol. 1. (Ed. R.C. Forzza). Rio de Janeiro: Andrea Jakobsson Estúdio; Instituto de Pesquisas Jardim Botânico do Rio de Janeiro. pp. 262-309.

Guiry, M.D. \& G.M. Guiry. 2012. AlgaeBase. World-wide electronic publication, National University of Ireland, Galway. http://www.algaebase.org; searched on 22 September, 2012.

Hickel, B. 1973. Limnological investigations in lakes of Pokhara valley, Nepal. Int. Rev. ges Hydrobiol. 58(5): 659-672.

Hirano, M. 1955. Fresh water algae. In: Fauna and flora of Nepal Himalaya (Ed. H. Kihara). Fauna and Flora Research Society, Kyoto University, Kyoto, Japan. pp. 5-42.

Hirano, M. 1969. Fresh water algae from Langtang Himal, Nepal Himalaya. Contr. Biol. Lab., Kyoto Univ., Japan. 22: 1-42.

Hu, H. \& Y. Wei. 2006. The freshwater algae of China. Systematics, taxonomy and ecology. China. pp. 1-1023. www.sciencep.com. Browse on $24^{\text {th }}$ Sept., 2012.

Ishida, Y. (Ed.) 1986. Studies on distribution, adaptation and evolution of microorganisms in Nepal Himalayas (Second report). Kyoto, Japan. pp. 3-13.

Jamaloo, F., F. Falahian, T. Nejadsatari \& A. Majd 2006. Study of diatoms flora in Jajrood river. Sciences and Technology of Environment 26: 98-112.

Misra, P.K., S.K. Rai \& M.N. Srivastava. 2009. Fresh water diatoms from eastern Nepal- I. Indian Hydrobiology 12(1): 95-104.

Nakanishi, M., A. Terashima, M. Watanabe \& P.N. Mishra. 1984. Preliminary report on limnological survey in lakes of the Pokhara valley (Nepal) in November-December 1982. In: Studies on distribution, adaptation and evolution of microorganisms in Nepal Himalayas (Preliminary Report) (Ed. H. Kadota). Kyoto, Japan. pp. 31-41.

Patrick, R. \& C.W. Reimer. 1966. The diatoms of the United States, exclusive of Alaska and Hawaii. Monograph of the Academy of Natural Sciences, Philadelphia, No. 13, Vol. I. $688 \mathrm{p}$.

Pérez, M.C, A. Comas \& N. Maidana 2010. Estudio taxonómico del fitoplancton del tramo inferior del río Júcar con especial énfasis en las algas verdes cocales (Valencia-España). ALGAS. Boletín de la Sociedad Española de Ficología 44: 13-19.

Rai, S.K. \& P.K. Misra. 2010. Freshwater cyanophyceae from east Nepal. Bangladesh J. of Plant Taxonomy 17(2): 121-139.

Rai, S.K. \& R.K. Rai. 2005. Some bacillariophycean algae from Biratnagar, Nepal. Ecoprint 12: 71-76.

Rai, S.K. 2005. Preliminary report of diatoms from Maipokhari lake, Ilam, Nepal. Our Nature 3(1): 26-30.

Rai, S.K. 2009. Some chlorophycean algae from Maipokhari Lake, Ilam, east Nepal. J. Nat. Hist. Mus, Nepal 24: 1-8.

Rai, S.K. 2011. Algal flora of Betana wetland, Morang, Nepal. Nepalese Journal of Biosciences 1: 104-113. 
Rai, S.K. 2011. Algal flora of Betana wetland, Morang, Nepal. Nepalese Journal of Biosciences 1: 104-113.

Rai, S.K., B.R. Subba \& K.P. Limbu. 2008. Fresh water algae from running streams of Gajurmukhi VDC, Ilam, Nepal. Our Nature 6: 80-81.

Rai, S.K., R.K. Rai \& N. Poudel. 2008. Desmids from Bees-hazaar lake, Chitwan, Nepal. Our Nature 6: 58-66.

Roberts, S., S. Sabater \& J. Beardall. 2004. Benthic microalgal colonization in streams of differing riparian cover and light availability. Journal of Phycology 40: 1004-1012.

Rodriguez, P.L., H. Pizarro, N. Maidana, Afonso Dos Santos \& S.M. Bonaventura. 2006. Epixylic algae from a polluted lowland river of Buenos Aires province (Argentina). Cryptogamie, Algologie 27: 63-83.

Sahay, A.P., P.K. Das \& B.N. Verma. 1992. Studies on the algal flora of Nepal- I: Chlorophyceae. Geophytology 20(2): 155-158.

Sherwood, A.R. 2004. Bibliographic checklist of the nonmarine algae of the Hawaiian Islands. Records of the Hawaii Biological Survey for 2003. Bishop Museum Occasional Papers 80: $1-26$.

Skuja, A. 1948. Taxonomie des Phytoplanktons einiger Seen in Uppland, Schweden. Symbolae Botanicae Upsalienses 9(3): 1-399.

Surana, R., B.R. Subba \& K.P. Limbu. 2010. Physico-chemical studies on Chimdi lake of Sunsari district during its restoration stage. Our Nature 8: 258-269.

Tsarenko, P.M., H. Lange-Bertalot, V.V. Stupina \& S.P. Wasser 2000. Bacillariophyta. In: Biodiversity of cyanoprocaryotes, algae and fungi of Israel. Cyanoprocaryotes and algae of continental Israel (Eds. E. Nevo \& S.P. Wasser). Ruggell: A.R.A. Gantner Verlag K.-G. pp. 195-335.

Vinogradova, O.M., S.P. Wasser \& E. Nevo 2000. Cyanoprocaryota. In: Biodiversity of cyanoprocaryotes, algae and fungi of Israel. Cyanoprocaryotes and algae of continental Israel (Eds. E. Nevo. \& S.P. Wasser). Ruggell: A.R.A. Gantner Verlag K.-G. pp. 32141.

Watanabe, M. 1995. Algae from Lake Rara and its vicinities, Nepal Himalayas. In: Cryptogams of the Himalayas, Vol. 3, Nepal and Pakistan (Eds. M. Watanabe \& H. Hagiwara). National Science Museum, Tsukuba, Japan. pp. 1-17.

Whitton, B.A., D.M. John, L.R. Johnson, P.N.G. Boulton, M.G. Kelly \& E.Y. Haworth. 1998. A coded list of freshwater algae of the British Isles. LOIS Publication number 222. Wallingford: Institute of Hydrology \& NERC. pp. 1-274. 